\title{
'If-then' planning in one-to-one behaviour change counselling is effective in promoting contraceptive adherence in teenagers
}

\author{
Jilly Martin, ${ }^{1}$ Pauline Slade, ${ }^{2}$ Paschal Sheeran, ${ }^{3}$ Alison Wright, ${ }^{4}$ Tracey Dibble ${ }^{4}$
}

\author{
${ }^{1} S c h o o l$ of Health and Social \\ Care, Oxford Brookes University, \\ Oxford, UK \\ ${ }^{2}$ Clinical Psychology Unit, \\ University of Sheffield, Sheffield \\ UK \\ ${ }^{3}$ Department of Psychology, \\ University of Sheffield, Sheffield, \\ UK \\ ${ }^{4}$ Sheffield Contraception and \\ Sexual Health Service, Central \\ Health Clinic, Sheffield, UK
}

\section{Correspondence to} Dr Jilly Martin, School of Health and Social Care, Oxford Brookes University, Jack Straws Lane, Marston, Oxford OX3 OFL, UK; jmartin@brookes.ac.uk

Received 1 March 2010 Accepted 25 August 2010

\begin{abstract}
Background Family planning clinicians were trained to provide one-to-one behaviour change counselling to deprived teenage women. They facilitated 'if-then' planning designed to increase adherence to contraception and to overcome potential barriers to adherence.

Methods Reasons for consultation at the clinic were obtained from electronic records and compared before and after the intervention. Results It was found that consultation for emergency contraception and pregnancy testing was reduced by $15 \%$ (49\% vs $34 \%$ ) after making if-then plans. This compared to a $4 \%$ reduction in a usual care comparison group. Changes in reasons for consultation before and after the intervention were also explored. Positive changes in behaviour were exhibited in $53 \%$ of the group who made ifthen plans compared to $28 \%$ of the usual care comparison group. Changes in reasons for consultation over time were significant in the counselling group ( $n=87, p=0.035)$ but not in the comparison group ( $n=79, p=0.68)$.

Conclusion These findings provide evidence that training clinicians in psychological behaviour change techniques such as if-then planning can be effective in influencing contraceptive consultation patterns among teenage women.
\end{abstract}

\section{Introduction}

The UK has the highest rates of under-18 pregnancy in Western Europe, ${ }^{1}$ although there is evidence of a recent downward trend, with overall national rates now being at their lowest in 20 years. $^{2}$ However, in some areas of the UK, teenage pregnancy rates have steadily increased ${ }^{3}$ despite the inception, from 1999, of teenage pregnancy strategy-related activities that aim to halve under-18 pregnancy rates by 2010. ${ }^{1}$ Teenage pregnancy in the UK not only has extensive economic impacts but has contributed to widening and perpetuating health inequalities, ${ }^{4}$ with half of all

\section{Key message points \\ 'If-then' planning is a useful strategy for changing pregnancy-risk behaviour in young women. \\ - Training family planning clinicians to incorporate if-then planning into contraceptive consultations with young women is effective in reducing consultations associated with pregnancy-risk behaviour. \\ Designing and conducting tailored individual-level interventions that are delivered by trained clinicians is likely to promote contraceptive adherence and impact upon pregnancy-risk behaviour and pregnancy rates.}

under-18 conceptions occurring in the $20 \%$ most deprived wards in the UK. ${ }^{3}$

Guidelines published by the National Institute for Health and Clinical Excellence $(\mathrm{NICE})^{5}$ recommend that one-to-one interventions based on psychological behaviour change theories are provided to young people by sexual health professionals to prevent sexually transmitted infections and under-18 conceptions in the UK. NICE encourage the development of a research agenda to determine exactly which psychological behaviour change theories, or theory components, will be effective in achieving this aim. Interventions evaluated so far have been largely unsuccessful in delaying sexual intercourse, increasing contraceptive use or reducing pregnancy rates, ${ }^{6}$ although evidence indicates that theoretical interventions that are tailored and delivered to a particular group of adolescents by trained facilitators are associated with a decrease in sexual risk behaviour. ${ }^{17}$

Forming implementation intentions, or 'if-then' plans, is a useful technique for changing individual behaviour. ${ }^{8}$ Forming if-then plans can help people use their contraception more reliably by linking a situation with a desired behaviour. For example, to achieve the goal of taking a contraceptive pill every day, one might link a critical situation in the 'if-'part of the plan (e.g. "If I am in the bathroom 
after brushing my teeth...") with a suitable behaviour in the 'then-'part of the plan (e.g. "then I will take my contraceptive pill!"). Formulating plans in this way involves spelling out in advance exactly when, where and how one will reach a goal so that once the situation is encountered, behaviour will automatically be triggered without the need for further decision making. ${ }^{9}$ A recent meta-analysis has shown that if-then planning has beneficial effects for a range of health behaviours. $^{10}$

Formulating if-then plans in a questionnaire has been found to be effective in reducing consultation at a family planning clinic (FPC) for emergency contraception (EC) and pregnancy testing (PT) among teenage women over a 9 -month period. ${ }^{11}$ However, NICE guidelines ${ }^{5}$ highlight the potential of the contraceptive consultation as a platform for delivering behaviour change interventions. In addition, clinical guidelines ${ }^{12}$ advise that adherence to contraception should be targeted as part of good communication between sexual health clinicians and young patients. The present study therefore aimed to extend the findings of Martin et al. ${ }^{11}$ and test the effectiveness of training sexual health clinicians to develop individually tailored if-then plans in a one-to-one contraceptive consultation with young women. It was predicted that making if-then plans would reduce consultations for EC and PT at a FPC.

\section{Methods}

\section{Design}

A longitudinal pre-/postintervention design was employed to test the impact of if-then planning on consultation for EC and PT. It was not possible to conduct a randomised control trial (RCT) due to the nature of the intervention, which involved changing the routine practice of family planning clinicians. When participants were recruited into the study, they formed ifthen plans during a contraceptive consultation with trained staff. Participants' reasons for consulting the FPC (contraceptive supplies, EC or PT) were recorded from electronic clinic notes for a 9-month baseline period (pre-intervention) and a 9-month follow-up period (post-intervention).

\section{Power}

Based on a medium effect size (0.03) for implementation intention interventions and to achieve a power of 0.08 for Chi-square $\left(\chi^{2}\right)$ frequency tests, a sample size of 88 was required.

\section{Setting}

This study was conducted in a UK National Health Service (NHS) FPC in a Northern UK city, which has higher than national average levels of deprivation and teenage conception rates. ${ }^{13} 14$ FPCs in the UK are widely used and offer free contraceptive advice and provision to young people who self-initiate a visit. Consultations are provided by doctors and nurses trained in family planning.

\section{Participants}

Participants were 87 young women aged 14-19 years visiting the FPC during the recruitment period (September-December 2007). Participants had a mean age of $16.8(\mathrm{SD}=1.52)$ years. Participants were recruited by 19 clinical staff (12 family planning nurses and seven doctors) who had completed the intervention training, plus a 2-week practice period. Participants were included in the study if they were attending the clinic for contraceptive advice/provision and were using a user-dependent method of contraception (i.e. injection, pill or condom). Participants were excluded from participation in the research if they (1) were attending the clinic requesting a referral for a termination, (2) had tested positive for pregnancy during the consultation or (3) were attending as a result of sexual assault.

\section{Implementation intention intervention}

A 1-day intervention training package was developed and delivered by the researchers. A practical strategy for delivery of the intervention was developed in consultation with clinicians, and an intervention protocol was produced that set out the procedure for delivering the intervention. During the course of a contraceptive consultation clinicians (1) introduced planning to young women, (2) identified a suitable target behaviour(s) for a plan, (3) developed a plan(s) by working through the when, where, how of the behaviour, (4) recorded and rehearsed the if-then plan and (5) provided positive feedback about the plan.

During intervention training, clinicians learnt the theoretical background of the intervention and were taught to identify the behaviours preceding successful contraceptive use, and the potential barriers that might prevent these behaviours being carried out. Interactive role plays and group feedback helped clinicians to practise and refine the techniques. Their role was to guide participants to develop personal if-then plans within contraceptive consultations that identified the best way to help them adhere to their contraceptive method by specifying a good situation in which to carry out the appropriate behaviour. Contraceptive pill users developed plans to help them take their pill every day (e.g. "If I am putting my mascara on in the morning before leaving the house, then I will take my pill from my make-up bag.") and condom users developed plans to address preparatory actions ${ }^{15}$ such as obtaining condoms, carrying condoms, or suggesting their use to a partner (e.g. "If I have only got one condom left, then I will go to the clinic on Thursday to get some more."). Where a potential barrier to adherence was identified during the consultation, such as a situation where a pill was often forgotten or encountering an unexpected barrier to condom use, a second plan was made to overcome this (e.g. "If I stay at my friends' house for the night, then I will remember to pack my pill along with my pyjamas" or "If my partner refuses to use a condom, then I will not have sex."). Clinicians asked participants to repeat 
the plan aloud to rehearse the if-then link, and gave the participant positive feedback about their plan.

\section{Outcome measures}

Electronic records of participants' visits to the FPC were assessed for the 9-month baseline and follow-up periods. Participants were allocated one of two codes based on the reason they visited the clinic: (1) visited for EC or PT or (2) visited for contraceptive supplies only.

\section{Results}

The effect of if-then planning on consultation outcomes over time was assessed by (1) comparing the reasons for consultation at baseline and follow-up periods and (2) exploring the changes in reasons for consultation between baseline and follow-up. As a further test of the intervention, comparisons were made to an equivalent group $(n=79)$ who had received usual care. Data from the usual care comparison group were collected approximately 1 year before the intervention (data published elsewhere). ${ }^{11}$

\section{Comparing reasons for consultation between baseline and follow-up}

Reasons for visiting the clinic at baseline and follow-up were compared. Table 1 illustrates a $15 \%$ reduction in consultation for EC/PT at follow-up $(34 \%, n=30)$ compared to baseline $(49 \%, n=43)$. This compares to a small non-significant reduction from $57 \%$ at baseline to $53 \%$ at follow-up in the usual care comparison group.

\section{Changes in reasons for consultation between baseline and follow-up}

Changes in the reasons for consultation between baseline and follow-up were explored (Table 2). For participants who visited the clinic at baseline for EC/PT $(n=43), 53 \%(n=23)$ made a positive change and visited at follow-up for supplies only. This was compared to only $23 \%(n=10)$ of participants who visited the clinic at baseline for supplies only $(n=44)$ and then made a negative change to visiting for EC/PT at follow-up. Within-group McNemar tests indicated that these changes over time were significant $(p=0.035)$. The corresponding figures in the usual care comparison group (28\% and $29 \%$, respectively) were not significant $(n=79, p=0.68)$.

\begin{tabular}{|c|c|c|c|c|}
\hline \multirow[b]{2}{*}{ Reason for consultation } & \multicolumn{2}{|c|}{ Baseline } & \multicolumn{2}{|c|}{ Follow-up } \\
\hline & $n$ & $\%$ & $n$ & $\%$ \\
\hline Supplies & 44 & 51 & 57 & 66 \\
\hline EC/PT & 43 & 49 & 30 & 34 \\
\hline Total & 87 & 100 & 87 & 100 \\
\hline
\end{tabular}

\section{Discussion}

This study shows that behaviour change counselling based on if-then planning is effective in reducing rates of consultation at a FPC for EC/PT among deprived teenage women. Consultations for EC/PT were reduced by $15 \%$ (49\% vs 34\%). Exploring the changes in reasons for consultation before and after the intervention showed that positive changes in behaviour were exhibited in 53\% of the group who made if-then plans compared to $28 \%$ of the usual care comparison group, and negative changes were made by $23 \%$ of the if-then planners, compared to $29 \%$ of the usual care comparison group. Changes in reasons for consultation over time were significant for the if-then planners but not the comparison group. These findings provide evidence that training clinicians in psychological behaviour change techniques such as ifthen planning can be effective in influencing contraceptive consultation patterns among teenage women.

The intervention reported here had theoretical and practical advantages: the one-to-one mode of delivery ensured that tailored if-then plans were made that targeted key behaviours to enhance individual contraceptive adherence. The present findings also provide evidence of the utility of if-then planning in everyday family planning practice and as a strategy for increasing the effectiveness of contraceptive counselling by employing a more flexible, women-centred approach to contraceptive education. ${ }^{16}$

There are a number of shortcomings to the present study. First, it was not possible to conduct an RCT to test this intervention as all trained clinicians in the FPC adopted the intervention technique as part of their routine practice. Thus, data for the usual care comparison group were collected before intervention training began to avoid contamination effects. There was no reason to believe that history effects caused the groups to differ systematically; however, data for both groups were collected over an identical time period to control for the impact of seasonal variations in sexual activity ${ }^{17}$ and the same clinicians were involved in the research at both time points. Nonetheless, future evaluations of this intervention should use an RCT design, across a number of sites, to provide a stronger test of if-then planning effects.

The second potential limitation concerns clinicians' fidelity to the intervention protocol. The delivery of the intervention was verified by the completion of a

Table 2 Changes between baseline and follow-up in reasons for consultation

\begin{tabular}{llll}
\hline \multirow{4}{*}{ Baseline } & \multicolumn{2}{l}{ Follow-up } & \\
\cline { 2 - 4 } & Supplies & EC/PT & Total \\
\hline Supplies & 34 & 10 & 44 \\
EC/PT & 23 & 20 & 43 \\
Total & 57 & 30 & 87 \\
\hline EC, emergency contraception; PT, pregnancy testing. &
\end{tabular}


custom-made stamp in the patient notes. However, it was not possible to ascertain whether the intervention was delivered exactly as it was intended. Standardising the content and delivery of complex interventions such as this is a major challenge ${ }^{18}$ and further tests of this intervention should build in fidelity checks to ensure consistent and homogenous delivery of the intervention to inform future replication. ${ }^{19}$

Despite the limitations discussed above, an if-then planning intervention had a positive impact on consultation for EC/PT over time in this preliminary study. Setting the research in a FPC has clear benefits for ecological validity and ensures that the findings are recognisable, relevant and can immediately transfer to everyday best practice in family planning. The findings clearly support NICE recommendations that training family planning clinicians in psychological behaviour change techniques may potentially reduce unintended pregnancy rates among deprived young women.

Acknowledgements The authors would like to acknowledge the facilitation work of Ann Burke, Sheffield Contraception and Sexual Health Service, in the organisation of staff training for this study.

\section{Competing interests None.}

Ethical approval The research was approved by North Sheffield NHS Research Ethics Committee.

\section{Provenance and peer review Not}

commissioned; externally peer reviewed.

\section{References}

1 Social Exclusion Unit. Teenage Pregnancy Strategy. London, UK: Social Exclusion Unit, 1999.

2 Teenage Pregnancy Independent Advisory Group. Annual Report 2008/09. London, UK: Department for Children, Schools \& Families, 2009.

3 Department for Education and Skills. Teenage Pregnancy Next Steps: Guidance for Local Authorities and Primary Care Trusts on Effective Delivery of Local Strategies. London, UK: Department for Education and Skills Publications, 2006.

4 Paranjothy S, Broughton H, Adappa R, et al. Teenage pregnancy: who suffers? Arch Dis Child 2009;94:239-245.
5 National Institute for Health and Clinical Excellence (NICE). Prevention of Sexually Transmitted Infections and Under 19 Conceptions. London, UK: NICE, 2007.

6 DiCenso A, Guyatt G, Willan A, et al. Interventions to reduce unintended pregnancies among adolescents: systematic review of randomised controlled trials. BMJ 2002;324:1426.

7 Robin L, Dittus P, Whitaker D, et al. Behavioral interventions to reduce incidence of HIV, STD, and pregnancy among adolescents: a decade in review. J Adolesc Health 2004;34:3-26.

8 Gollwitzer PM. Implementation intentions: strong effects of simple plans. Am Psychol 1999;54:493-503.

9 Brandstätter V, Lengfelder A, Gollwitzer PM. Implementation intentions and efficient action initiation.J Pers Soc Psychol 2001;81:946-960.

10 Gollwitzer PM, Sheeran P. Implementation intentions and goal achievement: a meta-analysis of effects and processes. Adv Exp Soc Psychol 2006;38:69-119.

11 Martin J, Sheeran P, Slade P, et al. Implementation intention formation reduces consultations for emergency contraception and pregnancy testing among teenage women. Health Psychol 2009;28:762-769.

12 Faculty of Family Planning and Reproductive Health Care Clinical Effectiveness Unit. FFPRHC Guidance (October 2004). Contraceptive choices for young people. J Fam Plann Reprod Health Care 2004;30:237-251.

13 Office of the Deputy Prime Minister. The English Indices of Deprivation 2004. London, UK: Office of the Deputy Prime Minister, 2004.

14 Office of National Statistics. Local Authority Under-18 Conception Statistics 1998-2006. London, UK: Office of National Statistics, 2008.

15 Abraham C, Sheeran P, Norman P, et al. When good intentions are not enough: modeling postdecisional cognitive correlates of condom use. J Appl Soc Psychol 1999;29:2591-2612.

16 Hayter M. The structure of contraceptive education and instruction within nurse led family planning clinics: a grounded theory study. J Clin Nurs 2009;18:2656-2667.

17 Wellings K, Macdowall W, Catchpole M, et al. Seasonal variations in sexual activity and their implications for sexual health promotion. J R Soc Med 1999;92:60-64.

18 Stephenson J, Imrie J. Why do we need randomised controlled trials to assess behavioural interventions? BMJ 1998;316:611-613.

19 Hardeman W, Michie S, Fanshawe T, et al. Fidelity of delivery of a physical activity intervention: predictors and consequences. Psychol Health 2008;23:11-24. 